\title{
Novel Technique for Number Plate Detection and Recognition
}

\author{
Aditya Sharma \\ M. Tech Scholar \\ Computer Science Engineering Department \\ IET Alwar
}

\author{
Deepak Chaudhary \\ Assistant Professor \\ Computer Science Engineering Department \\ IET Alwar
}

\begin{abstract}
In this work, it is been concluded that various techniques of car number plate recognization is reviewed. The Automatic number plate recognition (ANPR) is a mass reconnaissance strategy that utilizations optical character recognition on images to peruse the license plates on vehicles. They can utilize existing shut circuit television or street principle authorization cameras, or ones particularly designed for the errand. They are utilized by different police powers and as a strategy for electronic toll gathering on pay-per-use streets and observing movement action, for example, red light adherence in a convergence. ANPR can be utilized to store the images caught by the cameras and additionally the content from the license plate, with some configurable to store a photo of the driver. Among various proposed techniques morphological scanning technique is efficient technique to scan the whole image and extract number plate portion. The second efficient technique is split-and-merge segmentation to segment whole detected number plate. The segmented number plate is recognized using the neural networks. The discussed technique provides $91 \%$ accuracy of character reorganization.
\end{abstract}

Keywords

Number Plate Detection, image processing

\section{INTRODUCTION}

Image is a network, a cluster of components called components in the square frame which are organized as lines and sections. Image Processing is a procedure to change over an image into digital shape and perform a few operations to get an improved image and concentrate valuable information from it [1]. It is an investigation of any calculation that takes an image as info and returns an image as yield. Image processing is alluded to processing of a $2 \mathrm{D}$ picture by a PC. It is a type of sign benefit in which image is info like video frame or photo and is image or qualities connected with that image might be yield. Image processing framework regard images as two dimensional flags and set of signs processing strategies are connected to them. It is most recent advancements and its applications in different parts of a business [2]. Image Processing shapes center examination region inside designing and software engineering trains excessively.

\section{LICENSE PLATE RECOGNITION}

License plate recognition (LPR) is an image-processing innovation which is utilized to perceive vehicles by their license plates. This mastery is ahead of time prominence in security and activity establishments. License plate recognition framework is a use of computer vision. Computer vision is a technique for utilizing a computer to take out abnormal state information from a digital image. There are some futile homogeny among various license plates, for example, its measurement and the blueprint of the license plate. LPR framework comprise of the accompanying four phases [3]:

1. Image acquisition

2. License plate extraction

3. License plate segmentation

4. License plate recognition phases.

1. Image Acquisition: With the assistance of sensor image is caught and digitized it with the assistance of analog to digital convertor just when image is in analog structure utilized an image securing card that believers video signs to digital images in light of some equipment based image preprocessing. It comprises of three sections. These parts as follow:

Optical System: This is non-electronic part which is comprises of lenses and other comparative parts. Image input is given in this part. It misshapes the image.

Sensor: It is another vital part of the framework which changes optical sign to electrical proportionate.

Digitizer: In this section an analog electric comparable is changed to the digital adaptation inside two methodology first testing and quantization.

License Plate Extraction: License plate extraction is the most vital stage in a LPR framework [4]. This technique depends on scale shape examination, which in turn depends on the hypothesis that, characters have line-type shapes closeby and blob-type shapes all inclusive. Fit as a fiddle investigation, Gaussian filters at different parities blur the given image and bigger size shapes has rise at bigger scales. To see these scales the design of principal curvature plane is presented. By method for normalized principal curvatures, trademark focuses are separated from the scale space $x-y-t$. The position $(\mathrm{x}, \mathrm{y})$ demonstrates the position of the blueprint and the degree $t$ shows the inbuilt trademark measurement of relating figures. All these trademark calls attention to the hauling out of the shape from the set up image that has linetype shapes in the vicinity and blob-type shapes all around. At last the record of level and vertical line sections is aggregate and any rectangular locales orchestrating the measurements of a license plate are kept as candidate areas. The disservice of, this strategy is that it requires enormous memory and is computationally unreasonable.

3. License plate segmentation: The central thought after region developing is to remember one or more criteria that are quality for the sought region. In the wake of setting up the criteria, the image is scanned for any pixels that satisfy the necessities. At whatever point such a pixel is experienced, its neighbors are checked, and if any of the neighbors additionally parallel the criteria, both the pixels are measured as have a place with the same region. 
4. Recognition: It presents the strategies that were utilized to order and then perceive the individual characters. The characterization depends on the removed components. These elements are then characterized utilizing either the statistical, syntactic or neural methodologies.

\section{REVIEW OF LITERATURE}

R Shreyas, et.al (2017) proposed Automatic Number Plate Recognition (ANPR) System which is based on an image processing technology. The proposed system can be mainly used to monitor road traffic activities such as the identification of vehicle during traffic violations such as speed of vehicle and to detect at the street traffic signals lane violation. And thereby can be traced every vehicle for traffic rule violation and can provide the information to the concern authority to take further effective action, so we can have smooth traffic flow and also we can avoid accidents occurring on the traffic junction [5]. This system can also be used to assist the authorities in identifying for any stolen vehicle. The proposed system first detects for any vehicle which violates traffic rule and then captures the vehicle image. From the captured image using image segmentation technique the vehicle number plate region will be extracted. And the technique used for the character recognition on number plate is Optical character recognition. The system design also involves the design and development of GUI using Matlab, to ease the user in step by step recognizing the characters and numbers from the vehicle license plate and displaying on the desktop GUI screen.

Mahesh Babu K, et.al (2016) presented that for finding vehicles commonly used in field of security and safety system, LPDR plays a significant role and we need to recognize vehicles registration number at a certain distance. This paper has four major steps as follows: Preprocessing of captured image, Extracting license number plate region, Segmentation and Character Recognition of license plate. In pre-processing the desired vehicle image is taken through the digital camera, brightness of image is adjusted; noise removal using filters and image is converted to gray scale [6]. Exactions of license plate region consist of finding the edges in the image where exact location of licenses plate is located and crop it into rectangular frame. Segmentation plays a vital role in vehicle licenses plate recognition; the legibility of character recognition completely relies on the segmentation done. The approach which we have used is simple but appropriate. First we segmented all characters in the image (LP) using Bounding box method. Finally, recognition of each character is done. The template matching method is used for recognition each character in the vehicle license plate.

Bhavin V Kakani, et.al (2017) presented that significant research and development of algorithms in intelligent transportation has grabbed more attention in recent years. An automated, fast, accurate and robust vehicle plate recognition system has become need for traffic control and law enforcement of traffic regulations; and the solution is ANPR [7]. This paper is dedicated on an improved technique of OCR based license plate recognition using neural network trained dataset of object features. A blended algorithm for recognition of license plate is proposed and is compared with existing methods for improving accuracy. The whole system can be categorized under three major modules, namely License Plate Localization, Plate Character Segmentation, and Plate Character Recognition. The system is simulated on 300 national and international motor vehicle LP images and results obtained justify the main requirement.
Muhammad Attique Khan, et.al (2018) a novel system is proposed for LPR based on the features selection. The proposed system comprises of three major step: (i) preprocessing and segmentation of character area, (ii) features extraction of ROI, and (iii) features fusion using novel technique. With proposed method, we have tried our best to deal with different problems of light variation, occlusion, and so on [8]. The simulation results confirm that, with our novel idea, we have managed to tackle above mentioned problems. Moreover, it is also concluded that a cascaded design can comfortably manage mentioned problem at its early stages and also the selection of good features results in improved classification accuracy. As a future work, we will add few more set of features and will implement improved features selection technique to reduce the error rate and increase the recognition rate.

Moustafa M. Kurdi, et.al (2017) a license plate recognition technology will be addressed in regards to the following components: image preprocessing, segmentation and recognition of plates' numbers. This technology allows recognizing license plates efficiently with day and night time images. Automatic number plate recognition (ANPR) system is a part of intelligent transport systems due to its efficient applications in areas such as highway electronic toll collection, traffic monitoring system, parking management, parking fee and so on. The article will delineate an automatic (programmed) neural network recognition system of auto license plates that was connected in numerous nations and our capacity to utilize this framework on Lebanese auto tags. This system depends on the extraction of the license plate, segmentation (division) of characters [9],

J. Albert Mayan, et.al (2016) presented that in a diverse country like India, gated communities, corporate premises and university campuses witness a lot of unfamiliar vehicles with number plates in different formats, fonts, font sizes and sometimes even in various languages which enter and leave the estate every day, and it is difficult to register the vehicle numbers manually even for a multi-lingual person [10]. This document aims to extract image frames from a streaming CCTV footage, recognize the vehicle number and convert it into its corresponding text format. Template matching has been used earlier in recognition of digits and letters, this paper also uses the concept of template matching methodology using a SIMULINK model in MATLAB which is developed to extract the vehicle number from the number plate.

\section{RESEARCH METHODOLOGY}

To perceive number plate above all else includes templates from A-z and 0-9 and include them into mat file. After that read the image and change over that image into dim scale. Presently the following stride is to discover edge value of the image. In the wake of discovering T-value change over that image into binary. In this work, we likewise utilize median filter and Gabor filter in which we made cell in which distinctive - diverse subplot in view of pixel value and used following formula:

$$
4 * \mathrm{pi} / 5, \operatorname{sqrt}(2), 3 * \mathrm{pi} / 2
$$

All the words are store in the form of matrix from image. Then the loop is used to find out connected components. It will do line by line, sentence by sentence. After that, result will be executed. 


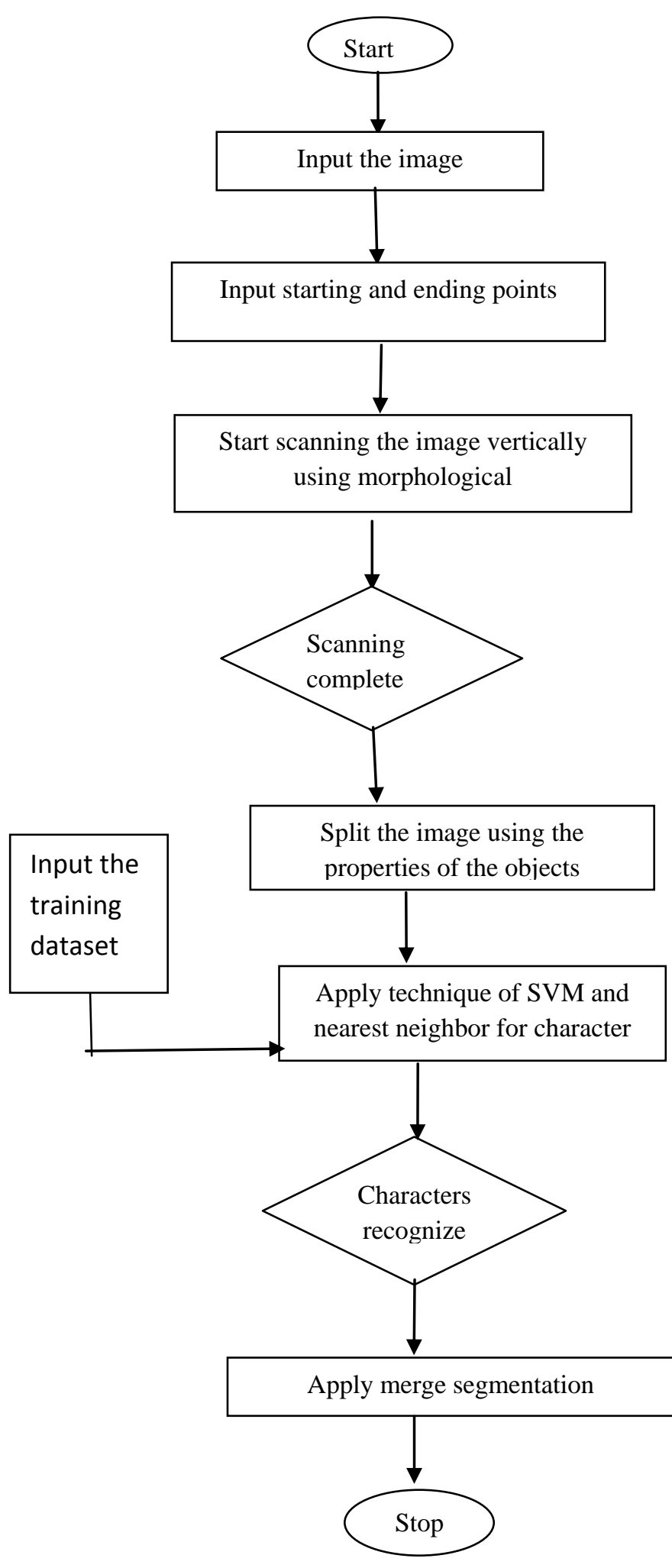

Fig: 1, Flowchart of proposed methodology

Read three images which having different angle with different blur, size etc.Then define path in which image can be take from same folder without read path of particular image.Then use the recognized start in which clock function used for time show how it takes. Further add path of all Matlab files in which there are two different functions which perform and then normalization is done.In this process there is a use of angle function in which if image at different angle than we recognize easily than regions define, then lines are drawn, rectangle for number is recognized according to their length, than draw sequence sketch according to their alphabet size. Then the region is drawn according to their sketch and this shows the regions. ).Acc to output LC length in which number plate than it draw red regions for easily recognize so other all parts cannot be detect or do not waste time to recognize, than its show different 2 times for LC normal methods. Another class of locally edge distinct locators taking into account extremely districts, which can be adjusted by machine learning systems to discretionary shapes, is proposed. In the test set of license plate images taken from various perspectives [-45, 45], scales (from seven to several pixels tallness) even in awful brightening conditions and partial impediments, the high detection precision is accomplished $(95 \%)$. At last we show the locator non specific capacities by movement signs detection. The standard classifier (neural network) inside the locator chooses a pertinent subset of extremely locales, i.e. areas that are associated segments of a threshold image. Properties of extremely areas render the finder exceptionally robust to brightening change and partial impediments. Robustness to a perspective change is accomplished by utilizing invariant descriptors and/or by demonstrating shape varieties by the classifier. The timemultifaceted nature of the detection is approximately direct in the number of pixel and a non-streamlined execution keeps running at around 1 outline for every second for a $640 \times 480$ image on a top of the line PC.

\section{RESULTS AND DISCUSSION}

The MATLAB is the tool which is used to implement proposed and existing algorithms. The results are analyzed in terms of various parameters. The proposed algorithm performs well in terms of all parameters

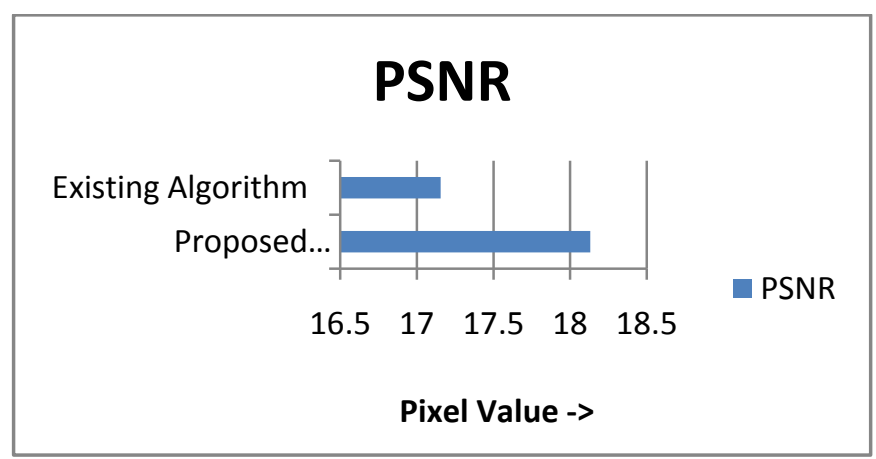

Fig 2, PSNR compression

As illustrated in Figure 2, the PSNR value of proposed algorithm is more than existing algorithm due to batter number plate detection in the system.

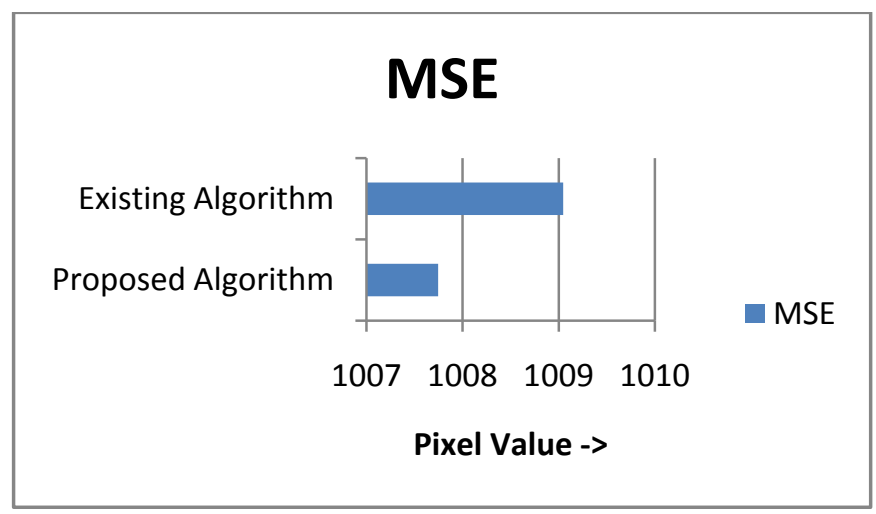

Fig 4.16: MSE Comparison 
As shown in Figure 4.16, the MSE value of the proposed algorithm is less as compared to proposed algorithm due use of nearest neighbor algorithm for detection of number plate.

\section{Fault Detection rate}

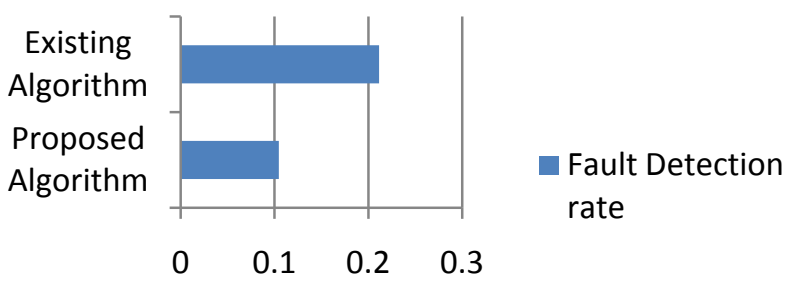

Pixels Not Detected

\section{Fig 4.17: Fault Detection rate}

As illustrated in Figure 4.17, the fault detection rate of proposed algorithm is less due to split and merge segmentation and use of nearest neighbor classifier in the algorithm.

\section{REFERENCES}

[1] Amar Badr Mohamed M. Abdelwahab, Ahmed M. Thabet, and Ahmed M.Abdelsadek, "Automatic Number Plate Recognition System", Annals of the University of Craiova, Mathematics and Computer Science Series Volume 38(1), 2011, Pages 62\{71ISSN: 1223-6934

[2] Anagnostopoulos, C.-N.; Anagnostopoulos, I.; Psoroulas, I.D.; Loumos, V.," License plate recognition from still images and video sequences: A survey", IEEE Intell. Transp. Syst. 2008, 9, 377-391

[3] Hamami, L., and, Berkani, D., "Recognition System for Printed Multi-Font and Multi-Size Arabic Characters", The Arabian Journal for Science and Engineering, vol. 27, no. IB, pp. 57-72, 2002
[4] Hansen, H., Kristensen, A. W., Kohler, M. P., Mikkelsen, A. W. ,Pedersen J. M., and Trangeled, M., "Automatic recognition of license plates", Institute for Electronic System, Aalhorg University, May 2002.

[5] R Shreyas, Pradeep Kumar B V, Adithya H B, Padmaja B, Sunil M P, "Dynamic Traffic Rule Violation Monitoring System Using Automatic Number Plate Recognition with SMS Feedback", 2017 2nd International Conference on Telecommunication and Networks (TEL-NET 2017)

[6] Mahesh Babu K, M V Raghunadh, "Vehicle Number Plate Detection and Recognition using Bounding Box Method", 2016 International Conference on Advanced Communication Control and Computing Technologies (ICACCCT)

[7] Bhavin V Kakani, Divyang Gandhi, Sagar Jani, "Improved OCR based Automatic Vehicle Number Plate Recognition using Features Trained Neural Network", 8th ICCCNT 2017

[8] Muhammad Attique Khan, Muhammad Sharif, Muhammad Younus Javed, Tallha Akram, Mussarat Yasmin, Tanzila Saba, "License number plate recognition system using entropy-based features selection approach with SVM", IET Image Process., 2018, Vol. 12 Iss. 2, pp. 200-209

[9] Moustafa M. Kurdi, Imad A. Elzein, Jalal Issa, Ibrahim Sayed Ahmad, "Lebanese Automated Number Plate Reading Based on Neural Network Recognition”, 2017, IEEE .

[10] Kumar, T. and K. Verma, 2010a. A theory based on conversion of RGB image to gray image. Int. J. Computer. Appli., 7: 5-12. DOI: 10.5120/1140-1493.

[11] J. Albert Mayan, Kumar Akash Deep, Mukesh Kumar, "Number Plate Recognition using Template Comparison for various fonts in MATLAB", 2016, IEEE 